MATHEMATICS OF COMPUTATION

Volume 69, Number 230, Pages 463-480

S 0025-5718(99)01106-0

Article electronically published on May 19, 1999

\title{
COMPUTATIONAL SCALES OF SOBOLEV NORMS WITH APPLICATION TO PRECONDITIONING
}

\author{
JAMES H. BRAMBLE, JOSEPH E. PASCIAK, AND PANAYOT S. VASSILEVSKI
}

\begin{abstract}
This paper provides a framework for developing computationally efficient multilevel preconditioners and representations for Sobolev norms. Specifically, given a Hilbert space $V$ and a nested sequence of subspaces $V_{1} \subset V_{2} \subset \ldots \subset V$, we construct operators which are spectrally equivalent to those of the form $\mathcal{A}=\sum_{k} \mu_{k}\left(Q_{k}-Q_{k-1}\right)$. Here $\mu_{k}, k=1,2, \ldots$, are positive numbers and $Q_{k}$ is the orthogonal projector onto $V_{k}$ with $Q_{0}=0$. We first present abstract results which show when $\mathcal{A}$ is spectrally equivalent to a similarly constructed operator $\widetilde{\mathcal{A}}$ defined in terms of an approximation $\widetilde{Q}_{k}$ of $Q_{k}$, for $k=1,2, \ldots$.

We show that these results lead to efficient preconditioners for discretizations of differential and pseudo-differential operators of positive and negative order. These results extend to sums of operators. For example, singularly perturbed problems such as $I-\epsilon \Delta$ can be preconditioned uniformly independently of the parameter $\epsilon$. We also show how to precondition an operator which results from Tikhonov regularization of a problem with noisy data. Finally, we describe how the technique provides computationally efficient bounded discrete extensions which have applications to domain decomposition.
\end{abstract}

\section{INTRODUCTION}

Multilevel subspace decompositions provide tools for the construction of preconditioners. One of the first examples of such a construction was provided in [3], where a simple additive multilevel operator (BPX) was developed for preconditioning second order elliptic boundary value problems. This preconditioner was defined in terms of a nested sequence of multilevel piecewise linear and continuous approximation spaces $V_{1} \subset V_{2} \subset \ldots \subset V_{J}$. The analysis of the BPX preconditioner involves the verification of norm equivalences of the form

$$
\|u\|_{H^{1}(\Omega)}^{2} \simeq \sum_{k=1}^{J} h_{k}^{-2}\left\|\left(Q_{k}-Q_{k-1}\right) u\right\|_{L^{2}(\Omega)}^{2}, \quad \text { for all } u \in V_{J} .
$$

The above norms are those corresponding to the Sobolev space $H^{1}(\Omega)$ and $L^{2}(\Omega)$ respectively; $Q_{k}$ denotes the $L^{2}(\Omega)$ orthogonal projection onto $V_{k}$ and $Q_{0}=0$. The quantity $h_{k}$ is the approximation parameter associated with $V_{k}$. The original

Received by the editor January 14, 1998 and, in revised form, June 23, 1998.

1991 Mathematics Subject Classification. Primary 65F10, 65N20, 65N30.

Key words and phrases. Interpolation spaces, equivalent norms, finite elements, preconditioning.

The first two authors were partially supported under National Science Foundation grant number DMS-9626567. The third author was partially supported by the Bulgarian Ministry for Education, Science and Technology under grant I-504, 1995. 
results in [3] were sharpened by [13] and [20 to show that (1.1) holds with constants of equivalence independent of $J$. Practical preconditioners involve the replacement of the operator $Q_{k}-Q_{k-1}$ by easily computable operators, as discussed in [3].

In addition to the above application, there are other practical applications of multilevel decompositions. In particular, for boundary element methods, it is important to have computationally simple operators which are equivalent to pseudodifferential operators of order one and minus one. In addition, multilevel decompositions which provide norm equivalences for $H^{1 / 2}(\partial \Omega)$ can be used to construct bounded extension operators used in nonoverlapping domain decomposition with inexact subdomain solves.

The equivalence (1.1) is the starting point of the multilevel analysis. This inequality is valid for $J=\infty$, in which case we get a norm equivalence on $H^{1}(\Omega)$. It follows from (1.1) that

$$
\|v\|_{H^{s}(\Omega)}^{2} \simeq \sum_{k=1}^{\infty} h_{k}^{-2 s}\left\|\left(Q_{k}-Q_{k-1}\right) v\right\|_{L^{2}(\Omega)}^{2},
$$

for $s \in[0,1]$. Here $\|\cdot\|_{H^{s}(\Omega)}$ denotes the norm on the Sobolev space $H^{s}(\Omega)$ of order $s$. This means that the operator

$$
\mathcal{A}^{s}=\sum_{k=1}^{\infty} h_{k}^{-2 s}\left(Q_{k}-Q_{k-1}\right)
$$

can be used in preconditioning applications. However, $\mathcal{A}^{s}$ is somewhat expensive to evaluate, since the evaluation of the projector $Q_{k}$ requires the solution of a Gram matrix problem. Thus, many researchers have sought computationally efficient operators which are equivalent to $\mathcal{A}^{s}$.

Some techniques for constructing such operators based on wavelet or waveletlike space decompositions are given by [5], [9], [10, [15], 16], [18, [19] and others. In the domain decomposition literature, extension operators that exploit multilevel decomposition were used in [4], [8], and [12.

In this paper, we construct simple multilevel decomposition preconditioning operators which can also be used to define norms equivalent to the usual norms on Sobolev spaces. Specifically, we develop computationally efficient operators which are uniformly equivalent to the more general operator

$$
\mathcal{A}_{J}=\sum_{k=1}^{J} \mu_{k}\left(Q_{k}-Q_{k-1}\right),
$$

where $1 \leq J \leq \infty$ and $\left\{\mu_{k}\right\}$ are positive constants. We start by proving an abstract theorem. Let $\left\{\widetilde{Q}_{k}\right\}$, with $\widetilde{Q}_{k}: V_{J} \rightarrow V_{k}$, be another sequence of linear operators. The theorem shows that the operators $\mathcal{A}_{J}$ and

$$
\widetilde{\mathcal{A}}_{J}=\sum_{k=1}^{J} \mu_{k}\left(\widetilde{Q}_{k}^{t}-\widetilde{Q}_{k-1}^{t}\right)\left(\widetilde{Q}_{k}-\widetilde{Q}_{k-1}\right)
$$

are spectrally equivalent under appropriate assumptions on the spaces $V_{k}$, the operators $\widetilde{Q}_{k}$ and the sequence $\left\{\mu_{k}\right\}$. Here $\widetilde{Q}_{k}^{t}$ is the adjoint of $\widetilde{Q}_{k}$. The abstract results are subsequently applied to develop efficient preconditioners when $\widetilde{Q}_{k}$ is defined in terms of a simple averaging operator. Some partial results involving the operator used here were stated by Nepomnyaschikh [12]. 
Because of the generality of the abstract results, they can be applied to preconditioning sums of operators. An example of this is the so-called "singularly perturbed" problem resulting from preconditioning parabolic time stepping problems, which leads to

$$
\mu_{k}=\left(\epsilon h_{k}^{-2}+1\right)^{-1} \text {. }
$$

Here $\epsilon$ is the time step size. Our results give rise to preconditioned systems with uniformly bounded condition numbers independent of the parameter $\epsilon$.

Note that an $L^{2}$-stable local basis for the spaces $\left\{\operatorname{Range}\left(Q_{k}-Q_{k-1}\right)\right\}$ is provided in [16. With such a construction it is possible to obtain preconditioners for the applications considered in this paper. However, our approach is somewhat simpler to implement. In addition, our abstract framework allows for easy application to other situations such as function spaces which are piecewise quadratic.

An outline of the remainder of the paper is as follows. Section 2 gives an abstract framework for norm approximation in a Hilbert space setting, along with an abstract theorem which provides equivalence estimates comparing (1.3) and (1.4). In Section 3 we give an example of a sequence $\left\{\widetilde{Q}_{k}\right\}$ of computationally efficient operators in the case of polygonal domains, and verify that they satisfy the hypotheses required for application of the abstract theory. In Section 4 we discuss some applications. Finally, the results of numerical experiments which illustrate the effectiveness of the preconditioners are reported in Section 5.

\section{A NORM EQUIVALENCE THEOREM}

In this section, we provide abstract conditions which imply the spectral equivalence of (1.3) and (1.4). We start by introducing the multilevel spaces. Let $V$ be a Hilbert space with inner product $(\cdot, \cdot)$. We assume that we are given a nested sequence of approximation subspaces,

$$
V_{1} \subset V_{2} \subset \ldots \subset V
$$

and that this sequence is dense in $V$. Let $\theta_{j}, j=1,2, \ldots$, be a non-decreasing sequence of positive real numbers. Define $H$ to be the subspace of $V$ such that the norm

$$
\|\| v \|=\left(\sum_{j=1}^{\infty} \theta_{j}\left\|\left(Q_{j}-Q_{j-1}\right) v\right\|^{2}\right)^{1 / 2}
$$

is finite. Here $\|\cdot\|$ denotes the norm in $V, Q_{j}$ for $j>0$ denotes the orthogonal projection onto $V_{j}$, and $Q_{0}=0$. Clearly, $H$ is a Hilbert space and $\left\{V_{k}\right\}$ is dense in $H$.

The following properties are obvious from the construction.

1. The "inverse inequality" holds for $V_{j}$, i.e.,

$$
\|\| v\|\| \leq \theta_{j}^{1 / 2}\|v\|, \quad \text { for all } v \in V_{j} .
$$

2. The "approximation property" holds for $V_{j}$, i.e.,

$$
\left\|\left(Q_{j}-Q_{j-1}\right) v\right\| \leq \theta_{j}^{-1 / 2}\|\mid v\| \|, \quad \text { for all } v \in H .
$$

As discussed in the introduction, the abstract results will be stated in terms of an additional sequence of "approximation" operators, $\widetilde{Q}_{k}: V \rightarrow V_{k}$ for $k>0$ and $\widetilde{Q}_{0}=0$. These operators are assumed to satisfy the following three conditions, for $k=1,2, \ldots$. 
1. An "approximation property": There exists a constant $C_{A}$ such that

$$
\left\|\left(Q_{k}-\widetilde{Q}_{k}\right) v\right\| \leq C_{A} \theta_{k}^{-1 / 2}\|\| v\|\|, \quad \text { for all } v \in H .
$$

2. Uniform coercivity of $\widetilde{Q}_{k}$ : There exists a $\delta>0$ such that

$$
\delta\left\|v_{k}\right\|^{2} \leq\left(\widetilde{Q}_{k} v_{k}, v_{k}\right), \quad \text { for all } v_{k} \in V_{k} .
$$

3. The range of $\widetilde{Q}_{k}^{t}$, the adjoint of $\widetilde{Q}_{k}$, is contained in $V_{k}$. This condition is equivalent to

$$
\widetilde{Q}_{k} Q_{k}=Q_{k} \widetilde{Q}_{k} .
$$

Remark 2.1. Let $\left\{\phi_{i}\right\}_{i=1}^{m}$ be a basis for $V_{k}$. It is not difficult to see that there exists $\left\{f_{i}\right\}_{i=1}^{m}$ with $f_{i} \in V$ such that

$$
\tilde{Q}_{k} v=\sum_{i=1}^{m}\left(v, f_{i}\right) \phi_{i} \quad \text { for all } v \in V .
$$

Then

$$
\tilde{Q}_{k}^{t} w=\sum_{i=1}^{m}\left(w, \phi_{i}\right) f_{i} \quad \text { for all } w \in V_{k} .
$$

Thus Condition 3 above holds if and only if $f_{i} \in V_{k}$, for $i=1, \ldots, m$.

The purpose of this section is to provide abstract conditions which guarantee that the symmetric operators $\mathcal{A}_{J}$ and $\widetilde{\mathcal{A}}_{J}$, defined respectively by (1.3) and (1.4), are spectrally equivalent. Let $\mathcal{L}=\left(\ell_{k, j}\right)$ be the lower triangular (infinite) matrix with nonzero entries

$$
\ell_{k, j}=\left(\frac{\theta_{j} \mu_{k}}{\theta_{k} \mu_{j}}\right)^{1 / 2}, \quad k \geq j
$$

We assume that $\mathcal{L}$ has bounded $l_{2}$ norm, i.e.,

$$
\|\mathcal{L}\|_{\ell_{2}} \equiv \sup _{\left\{\xi_{k}\right\},\left\{\zeta_{k}\right\}} \frac{\sum_{k=1}^{\infty} \sum_{j \leq k} \ell_{k, j} \xi_{k} \zeta_{j}}{\left(\sum_{k=1}^{\infty} \xi_{k}^{2}\right)^{1 / 2}\left(\sum_{k=1}^{\infty} \zeta_{k}^{2}\right)^{1 / 2}} \leq C_{\mathcal{L}} .
$$

The above condition implies that

$$
\mu_{k} \leq C \theta_{k}
$$

for $C=C_{\mathcal{L}}^{2} \mu_{1} / \theta_{1}$. Thus, $\left(\mathcal{A}_{J} v, v\right)<\infty$ for all $v \in H$.

We introduce one final condition: There exists a constant $\alpha$ such that

$$
\mu_{k}+\mu_{k+1} \leq \alpha \mu_{k}, \quad \text { for } k=1,2, \ldots
$$

We can now state the main abstract theorem.

Theorem 2.1. Assume that conditions (2.3)-2.5), (2.7), and (2.8) are satisfied. Then the operator $\widetilde{\mathcal{A}}_{J}$ defined by (1.4), with $1 \leq J \leq \infty$, satisfies

$$
\begin{aligned}
& {\left[3\left(1+\alpha \delta^{-2} C_{A}^{2} C_{\mathcal{L}}^{2}\right)\right]^{-1}\left(\mathcal{A}_{J} v, v\right) \leq\left(\widetilde{\mathcal{A}}_{J} v, v\right)} \\
& \quad \leq 3\left(1+\alpha C_{A}^{2} C_{\mathcal{L}}^{2}\right)\left(\mathcal{A}_{J} v, v\right), \quad \text { for all } v \in H .
\end{aligned}
$$

Remark 2.2. If $W$ is the completion of $H$ under the norm $\|v\|_{\mathcal{A}}=\left(\mathcal{A}_{\infty} v, v\right)^{1 / 2}$, then the estimate of Theorem 2.1 extends to all of $W$ by density. 
For the purpose of proving the theorem, we now prove the following lemma.

Lemma 2.1. Assume that conditions (2.3)-(2.5), and 2.7) are satisfied. Then, for all $u \in H$,

$$
\sum_{k=1}^{J} \mu_{k}\left\|\left(Q_{k}-\widetilde{Q}_{k}\right) u\right\|^{2} \leq C_{A}^{2} C_{\mathcal{L}}^{2}\left(\mathcal{A}_{J} u, u\right)
$$

and

$$
\sum_{k=1}^{J} \mu_{k}\left\|\left(Q_{k}-\widetilde{Q}_{k}\right) u\right\|^{2} \leq \delta^{-2} C_{A}^{2} C_{\mathcal{L}}^{2}\left(\widetilde{\mathcal{A}}_{J} u, u\right) .
$$

Proof. By (2.5), for all $u \in H$,

$$
\begin{gathered}
\sum_{k=1}^{J} \mu_{k}\left\|\left(Q_{k}-\widetilde{Q}_{k}\right) u\right\|^{2}=\sum_{k=1}^{J} \mu_{k}\left(\left(Q_{k}-\widetilde{Q}_{k}\right) Q_{k} u,\left(Q_{k}-\widetilde{Q}_{k}\right) u\right) \\
=\sum_{k=1}^{J} \sum_{j=1}^{k} \mu_{k}\left(\left(Q_{k}-\widetilde{Q}_{k}\right)\left(Q_{j}-Q_{j-1}\right) u,\left(Q_{k}-\widetilde{Q}_{k}\right) u\right) .
\end{gathered}
$$

In addition, by (2.4) and (2.5),

$$
\begin{aligned}
& \sum_{k=1}^{J} \mu_{k}\left\|\left(Q_{k}-\widetilde{Q}_{k}\right) u\right\|^{2} \leq \delta^{-1} \sum_{k=1}^{J} \mu_{k}\left(\widetilde{Q}_{k}\left(Q_{k}-\widetilde{Q}_{k}\right) u,\left(Q_{k}-\widetilde{Q}_{k}\right) u\right) \\
& \quad=\delta^{-1} \sum_{k=1}^{J} \mu_{k}\left(\left(Q_{k}-\widetilde{Q}_{k}\right) \widetilde{Q}_{k} u,\left(Q_{k}-\widetilde{Q}_{k}\right) u\right) \\
& =\delta^{-1} \sum_{k=1}^{J} \sum_{j=1}^{k} \mu_{k}\left(\left(Q_{k}-\widetilde{Q}_{k}\right)\left(\widetilde{Q}_{j}-\widetilde{Q}_{j-1}\right) u,\left(Q_{k}-\widetilde{Q}_{k}\right) u\right) .
\end{aligned}
$$

The quantities on the right hand side of (2.11) and of (2.12) can be written as

$$
\sum_{k=1}^{J} \sum_{j=1}^{k} \mu_{k}\left(\left(Q_{k}-\widetilde{Q}_{k}\right) v_{j},\left(Q_{k}-\widetilde{Q}_{k}\right) u\right)
$$

by setting $v_{j}$ equal to $\left(Q_{j}-Q_{j-1}\right) u$ and $\left(\widetilde{Q}_{j}-\widetilde{Q}_{j-1}\right) u$, respectively. Using (2.1) and (2.3), the quantity (2.13) is bounded by

$$
\begin{aligned}
\sum_{k=1}^{J} \sum_{j=1}^{k} \mu_{k}\left(\left(Q_{k}-\widetilde{Q}_{k}\right) v_{j},\left(Q_{k}-\widetilde{Q}_{k}\right) u\right) \\
\leq C_{A} \sum_{k=1}^{J} \sum_{j=1}^{k} \mu_{k} \theta_{k}^{-1 / 2}\left\|\mid v_{j}\right\|\|\|\left(Q_{k}-\widetilde{Q}_{k}\right) u \| \\
\leq C_{A} \sum_{k=1}^{J} \sum_{j=1}^{k} \mu_{k}\left(\theta_{j} / \theta_{k}\right)^{1 / 2}\left\|v_{j}\right\|\left\|\left(Q_{k}-\widetilde{Q}_{k}\right) u\right\| \\
\quad=C_{A} \sum_{k=1}^{J} \sum_{j=1}^{k} \ell_{k, j}\left(\mu_{j}^{1 / 2}\left\|v_{j}\right\|\right)\left(\mu_{k}^{1 / 2}\left\|\left(Q_{k}-\widetilde{Q}_{k}\right) u\right\|\right) .
\end{aligned}
$$


It immediately follows from (2.7) that

$$
\begin{aligned}
& \sum_{k=1}^{J} \sum_{j=1}^{k} \mu_{k}\left(\left(Q_{k}-\widetilde{Q}_{k}\right) v_{j},\left(Q_{k}-\widetilde{Q}_{k}\right) u\right) \\
& \quad \leq C_{A} C_{\mathcal{L}}\left(\sum_{k=1}^{J} \mu_{k}\left\|v_{k}\right\|^{2}\right)^{1 / 2}\left(\sum_{k=1}^{J} \mu_{k}\left\|\left(Q_{k}-\widetilde{Q}_{k}\right) u\right\|^{2}\right)^{1 / 2} .
\end{aligned}
$$

Combining (2.11) and 2.15) gives

$$
\begin{aligned}
& \sum_{k=1}^{J} \mu_{k}\left\|\left(Q_{k}-\widetilde{Q}_{k}\right) u\right\|^{2} \\
& \quad \leq C_{A} C_{\mathcal{L}}\left(\sum_{k=1}^{J} \mu_{k}\left\|\left(Q_{k}-Q_{k-1}\right) u\right\|^{2}\right)^{1 / 2}\left(\sum_{k=1}^{J} \mu_{k}\left\|\left(Q_{k}-\widetilde{Q}_{k}\right) u\right\|^{2}\right)^{1 / 2} .
\end{aligned}
$$

The inequality (2.9) follows by obvious manipulations, and (2.10) follows in a similar manner.

Proof of Theorem [2.1] Note that

$$
\left(\widetilde{Q}_{k}-\widetilde{Q}_{k-1}\right)=\left(Q_{k}-Q_{k-1}\right)-\left(Q_{k}-\widetilde{Q}_{k}\right)+\left(Q_{k-1}-\widetilde{Q}_{k-1}\right) .
$$

Thus, for $v \in H$,

$$
\begin{aligned}
\left(\widetilde{\mathcal{A}}_{J} v, v\right) & =\sum_{k=1}^{J} \mu_{k}\left\|\left(\widetilde{Q}_{k}-\widetilde{Q}_{k-1}\right) v\right\|^{2} \\
& \leq 3\left(\sum_{k=1}^{J} \mu_{k}\left\|\left(Q_{k}-Q_{k-1}\right) v\right\|^{2}+\sum_{k=1}^{J}\left(\mu_{k}+\mu_{k+1}\right)\left\|\left(Q_{k}-\widetilde{Q}_{k}\right) v\right\|^{2}\right) \\
& \leq 3\left(1+\alpha C_{A}^{2} C_{\mathcal{L}}^{2}\right)\left(\mathcal{A}_{J} v, v\right) .
\end{aligned}
$$

We used (2.8) and Lemma 2.1 for the last inequality above. The proof for the other inequality is essentially the same. This completes the proof of the theorem.

2.1. Development of preconditioners. The above results can be applied to the development of preconditioners. Indeed, consider preconditioning an operator on $V_{J}$ which is spectrally equivalent to

$$
L_{J}=\sum_{k=1}^{J} \mu_{k}^{-1}\left(Q_{k}-Q_{k-1}\right)
$$

Our preconditioner $B_{J}$ is to be spectrally equivalent to the operator

$$
A_{J} \equiv L_{J}^{-1}=\sum_{k=1}^{J} \mu_{k}\left(Q_{k}-Q_{k-1}\right)
$$

Let

$$
B_{J}=\sum_{k=1}^{J} \mu_{k}\left(\widetilde{Q}_{k}-\widetilde{Q}_{k-1}\right)^{t}\left(\widetilde{Q}_{k}-\widetilde{Q}_{k-1}\right) .
$$

Then $B_{J}$ and $A_{J}$ are spectrally equivalent provided that $\left\{\mu_{k}\right\}$ and $\left\{\widetilde{Q}_{k}\right\}$ satisfy the hypothesis of the theorem. It follows that $B_{J} L_{J}$ is well conditioned. 
2.2. Preconditioning sums of operators. We next consider the case of preconditioning sums of operators. Suppose $\left\{\hat{\mu}_{k}\right\}$ is another sequence which satisfies conditions (2.7) and (2.8). Then the operator

$$
\hat{L}_{J}=\sum_{k=1}^{J} \hat{\mu}_{k}^{-1}\left(Q_{k}-Q_{k-1}\right)
$$

can be preconditioned by the operator defined by replacing $\mu_{k}$ by $\hat{\mu}_{k}$ in (2.17) above. The following corollary shows that the result can be extended to nonnegative combinations of $L_{J}$ and $\hat{L}_{J}$.

Corollary 2.1. Assume that conditions 2.3)-2.5) are satisfied and that (2.7) and (2.8) hold for both $\left\{\mu_{k}\right\}$ and $\left\{\hat{\mu}_{k}\right\}$. For nonnegative $c_{1}, c_{2}$ with $c_{1}+c_{2}>0$ define

$$
B_{J}=\sum_{k=1}^{J}\left(c_{1} \mu_{k}^{-1}+c_{2} \hat{\mu}_{k}^{-1}\right)^{-1}\left(\widetilde{Q}_{k}-\widetilde{Q}_{k-1}\right)^{t}\left(\widetilde{Q}_{k}-\widetilde{Q}_{k-1}\right) .
$$

Then, for $1 \leq J \leq \infty$,

$$
\begin{array}{r}
{\left[3\left(1+4 \alpha \delta^{-2} C_{A}^{2} C_{\mathcal{L}}^{2}\right)\right]^{-1}\left(\left(c_{1} L_{J}+c_{2} \hat{L}_{J}\right)^{-1} v, v\right) \leq\left(B_{J} v, v\right)} \\
\leq 3\left(1+4 \alpha C_{A}^{2} C_{\mathcal{L}}^{2}\right)\left(\left(c_{1} L_{J}+c_{2} \hat{L}_{J}\right)^{-1} v, v\right), \quad \text { for all } v \in H .
\end{array}
$$

The above corollary shows that $B_{J}$ is spectrally equivalent to $\left(c_{1} L_{J}+c_{2} \hat{L}_{J}\right)^{-1}$ and hence provides a uniform preconditioner for $c_{1} L_{J}+c_{2} \hat{L}_{J}$. Moreover, the resulting condition number (for the preconditioned system) is bounded independently of the parameters $c_{1}$ and $c_{2}$.

Proof. Note that

$$
\left(c_{1} L_{J}+c_{2} \hat{L}_{J}\right)^{-1}=\sum_{k=1}^{J}\left(c_{1} \mu_{k}^{-1}+c_{2} \hat{\mu}_{k}^{-1}\right)^{-1}\left(Q_{k}-Q_{k-1}\right) .
$$

To apply the theorem to this operator, we simply must check the conditions on the sequence $\widetilde{\mu}_{k}=\left(c_{1} \mu_{k}^{-1}+c_{2} \hat{\mu}_{k}^{-1}\right)^{-1}$. The corresponding lower triangular matrix has entries

$$
\begin{aligned}
(\widetilde{\mathcal{L}})_{k, j} & =\left(\frac{\theta_{j} \widetilde{\mu}_{k}}{\theta_{k} \widetilde{\mu}_{j}}\right)^{1 / 2}=\left(\frac{\theta_{j}\left(c_{1} \mu_{j}^{-1}+c_{2} \hat{\mu}_{j}^{-1}\right)}{\theta_{k}\left(c_{1} \mu_{k}^{-1}+c_{2} \hat{\mu}_{k}^{-1}\right)}\right)^{1 / 2} \\
& \leq\left(\frac{\theta_{j}}{\theta_{k}}\left(\frac{\mu_{k}}{\mu_{j}}+\frac{\hat{\mu}_{k}}{\hat{\mu}_{j}}\right)\right)^{1 / 2} \leq\left(\frac{\theta_{j} \mu_{k}}{\theta_{k} \mu_{j}}\right)^{1 / 2}+\left(\frac{\theta_{j} \hat{\mu}_{k}}{\theta_{k} \hat{\mu}_{j}}\right)^{1 / 2}=(\mathcal{L}+\hat{\mathcal{L}})_{k, j} .
\end{aligned}
$$

Since $0 \leq(\widetilde{\mathcal{L}})_{k, j} \leq(\mathcal{L}+\hat{\mathcal{L}})_{k, j}$, for every pair $k, j$, it follows that

$$
\|\widetilde{\mathcal{L}}\|_{\ell_{2}} \leq\|\mathcal{L}+\hat{\mathcal{L}}\|_{\ell_{2}} \leq 2 C_{\mathcal{L}}
$$

Because (2.8) holds for both $\left\{\mu_{k}\right\}$ and $\left\{\hat{\mu}_{k}\right\}$, it clearly holds for $\left\{\widetilde{\mu}_{k}\right\}$. The corollary follows by application of the theorem.

\section{A Simple approximation operator $\widetilde{Q}_{k}$}

In this section, we define and analyze a simple approximation operator $\widetilde{Q}_{k}$. Our applications involve Sobolev spaces with possibly mixed boundary conditions.

Let $\Omega$ be a polygonal domain in $R^{2}$ with boundary $\partial \Omega=\Gamma_{D} \cup \Gamma_{N}$, where $\Gamma_{D}$ and $\Gamma_{N}$ are essentially disjoint. Dirichlet boundary conditions are imposed on $\Gamma_{D}$. We 
consider domains in $R^{2}$ for convenience. Generalizations of the results to domains in $R^{d}$, with $d>2$, at least for rectangular parallelepipeds, are straightforward.

For non-negative integers $s$, let $H^{s}(\Omega)$ denote the Sobolev space of order $s$ on $\Omega$ (see, e.g. [6],[7]). The corresponding norm and semi-norm are denoted $\|\cdot\|_{H^{s}(\Omega)}$ and $|\cdot|_{H^{s}(\Omega)}$ respectively. The space $H_{D}^{1}(\Omega)$ is defined to be the functions in $H^{1}(\Omega)$ which vanish on $\Gamma_{D}$, and, for $s>1, H_{D}^{s}(\Omega)=H^{s}(\Omega) \cap H_{D}^{1}(\Omega)$. For positive non-integers $s$, the spaces $H^{s}(\Omega)$ and $H_{D}^{s}(\Omega)$ are defined by interpolation between the neighboring integers using the real method of Lions and Peetre (cf. [7]). For negative $s, H^{s}(\Omega)$ is defined to be the space of linear functionals for which the norm

$$
\|u\|_{H^{s}(\Omega)}=\sup _{\phi \in H_{D}^{-s}(\Omega)} \frac{\langle u, \phi\rangle}{\|\phi\|_{H_{D}^{-s}(\Omega)}}
$$

is finite. Here $\langle\cdot, \cdot\rangle$ denotes the duality pairing. Clearly, for $s<0$ we have $L^{2}(\Omega) \subseteq$ $H^{s}(\Omega)$ if we identify $u \in L^{2}(\Omega)$ with the functional $\langle u, \phi\rangle \equiv(u, \phi)$.

3.1. Some basic approximation properties. Let $\mathcal{T}$ be a locally quasi-uniform triangulation of $\Omega$, and let $\tau$ be a closed triangle in $\mathcal{T}$ with diameter $h_{\tau}$. Let $\widetilde{\tau}$ be the subset of the triangles in $\mathcal{T}$ whose boundaries intersect $\tau$, and define $V_{\widetilde{\tau}}$ to be the finite element approximation subspace consisting of functions which are continuous on $\widetilde{\tau}$ and piecewise linear with respect to the triangles of $\widetilde{\tau}$. Note that there are no boundary conditions imposed on the elements of $V_{\widetilde{\tau}}$. We restrict the discussion in this paper to piecewise linear subspaces. Extensions to more general nodal finite element subspaces pose no significant additional difficulties.

The following facts are well known.

1. Given $u \in H^{1}(\widetilde{\tau})$, there exists a constant $\widetilde{u}$ such that

$$
\|u-\widetilde{u}\|_{H^{s}(\widetilde{\tau})} \leq C h_{\tau}^{1-s}|u|_{H^{1}(\widetilde{\tau})}, \quad s=0,1 .
$$

2. Given $u \in H^{2}(\widetilde{\tau})$, there exists a linear function $\widetilde{u}$ such that

$$
\|u-\widetilde{u}\|_{H^{s}(\widetilde{\tau})} \leq C h_{\tau}^{2-s}|u|_{H^{2}(\widetilde{\tau})}, \quad s=0,1,2 .
$$

The best constants satisfying the above inequalities clearly depend on the shape of the domain $\widetilde{\tau}$. However, under the assumption that the triangulation is locally quasi-uniform, it is possible to show that the above inequalities hold with constants only depending on $s$ and on the quasi-uniformity constants.

For the purpose of analyzing our multilevel example we define the following local approximation operator $\widetilde{Q}_{\tilde{\tau}}: L^{2}(\Omega) \rightarrow V_{\tilde{\tau}}$. Let $\phi_{i}, i=1,2, \ldots, m$, be the nodal basis for $V_{\widetilde{\tau}}$. The operator $\widetilde{Q}_{\widetilde{\tau}}$ is given by

$$
\widetilde{Q}_{\tilde{\tau}} u=\sum_{i=1}^{m} \frac{\left(u, \phi_{i}\right)_{\tilde{\tau}}}{\left(1, \phi_{i}\right)_{\tilde{\tau}}} \phi_{i},
$$

with $(\cdot, \cdot)_{\tilde{\tau}}$ the inner product in $L^{2}(\widetilde{\tau})$. For $u, v \in L^{2}(\widetilde{\tau})$ we have

$$
\left(\widetilde{Q}_{\tilde{\tau}} u, v\right)_{\tilde{\tau}}=\sum_{i=1}^{m} \frac{\left(u, \phi_{i}\right)_{\tilde{\tau}}\left(v, \phi_{i}\right)_{\tilde{\tau}}}{\left(1, \phi_{i}\right)_{\tilde{\tau}}}
$$

and hence it immediately follows that $\widetilde{Q}_{\widetilde{\tau}}$ is symmetric on $L^{2}(\widetilde{\tau})$. Moreover, $\widetilde{Q}_{\widetilde{\tau}}$ is positive definite when restricted to $V_{\widetilde{\tau}}$ (see Lemma 3.4). The next lemma provides a basic approximation property for $\widetilde{Q}_{\tilde{\tau}}$. 
Lemma 3.1. Let $\tau$ be in $\mathcal{T}$. Then for $s=0,1$, there exists a constant $C$, independent of $\tau$, such that

$$
\left\|u-\widetilde{Q}_{\tilde{\tau}} u\right\|_{L^{2}(\widetilde{\tau})} \leq C h_{\tau}^{s}\|u\|_{H^{s}(\widetilde{\tau})}, \quad \text { for all } u \in H^{s}(\widetilde{\tau}) .
$$

Proof. A simple computation shows that

$$
\left\|\widetilde{Q}_{\tilde{\tau}} u\right\|_{L^{2}(\widetilde{\tau})} \leq C\|u\|_{L^{2}(\widetilde{\tau})}
$$

from which (3.4) immediately follows for $s=0$. For $s=1$, let $\widetilde{u}$ be the constant function satisfying (3.1). Using the previous estimate, since $\widetilde{Q} \widetilde{\tau} \widetilde{u}=\widetilde{u}$, we have

$$
\left\|u-\widetilde{Q}_{\widetilde{\tau}} u\right\|_{L^{2}(\widetilde{\tau})} \leq\|u-\widetilde{u}\|_{L^{2}(\widetilde{\tau})}+\left\|\widetilde{Q}_{\widetilde{\tau}}(u-\widetilde{u})\right\|_{L^{2}(\widetilde{\tau})} \leq C\|u-\widetilde{u}\|_{L^{2}(\widetilde{\tau})} .
$$

Combining the above inequalities and (3.1) completes the proof of (3.4) for $s=$ 1.

3.2. Approximation properties: the multilevel case. We provide stronger approximation properties in the case when the mesh results from a multilevel refinement strategy. Again we describe the case of $d=2$. The analogous constructions for $d>2$, at least for the case of rectangular parallelepipeds, are straightforward generalizations. Assume that an initial coarse triangulation $\mathcal{T}_{1}$ of $\Omega$ has been provided with $\Gamma_{D}$ aligning with the mesh $\mathcal{T}_{1}$. By this we mean that any edge of $\mathcal{T}_{1}$ on $\partial \Omega$ is either contained in $\Gamma_{D}$ or intersects $\Gamma_{D}$ at most at the endpoints of the edge. Multilevel triangulations are defined recursively. For $k>1$, the triangulation $\mathcal{T}_{k}$ is defined by breaking each triangle in $\mathcal{T}_{k-1}$ into four, by connecting the centers of the edges. The finite element space $V_{k}$ consists of the functions which are continuous on $\Omega$, piecewise linear with respect to $\mathcal{T}_{k}$ and vanish on $\Gamma_{D}$. Let $h_{k}=\max _{\tau \in \mathcal{T}_{k}} h_{\tau}$. Clearly, $h_{k}=2^{-k+1} h_{1}$.

We now define a sequence of approximation operators $\widetilde{Q}_{k}: L^{2}(\Omega) \rightarrow V_{k}$. Let $\phi_{i}$, $i=1, \ldots, m$, be the nodal basis for $V_{k}$. We define $\widetilde{Q}_{k}$ by

$$
\widetilde{Q}_{k} u=\sum_{i=1}^{m} \frac{\left(u, \phi_{i}\right)}{\left(1, \phi_{i}\right)} \phi_{i} \text {. }
$$

Remark 3.1. Let $\tau$ be a triangle of $\mathcal{T}_{k}$. It is easy to see that $\widetilde{Q}_{\widetilde{\tau}} u$ and $\widetilde{Q}_{k} u$ agree on $\tau$ as long as $\tau \cap \Gamma_{D}=\emptyset$.

In the multilevel case, we have the following stronger version of Lemma 3.1

Lemma 3.2. Let $s$ be in $[0,3 / 2)$. There exists a constant $C_{s}$, not depending on $h_{k}$, such that

$$
\left\|u-\widetilde{Q}_{k} u\right\|_{L^{2}(\Omega)} \leq C_{s} h_{k}^{s}\|u\|_{H^{s}(\Omega)}, \quad \text { for all } u \in H_{D}^{s}(\Omega) .
$$

For the proof of the lemma we will use the following lemma, which is a slight modification of Lemma 6.1 of [1]. Its proof is contained in the proof of Lemma 6.1 of [1].

Lemma 3.3. Let $\Omega^{\eta}$ denote the strip $\{x \in \Omega \mid \operatorname{dist}(x, \partial \Omega)<\eta\}$, and let $0 \leq s<$ $1 / 2$. Then, for all $v \in H^{1+s}(\Omega)$,

$$
\|v\|_{H^{1}\left(\Omega^{\eta}\right)} \leq C \eta^{s}\|v\|_{H^{1+s}(\Omega)} .
$$

In addition, let $\Omega_{D}^{\eta}$ denote the strip $\left\{x \in \Omega \mid \operatorname{dist}\left(x, \Gamma_{D}\right)<\eta\right\}$. Then, for all $v$ in $H_{D}^{1}(\Omega)$,

$$
\|v\|_{L^{2}\left(\Omega_{D}^{\eta}\right)} \leq C \eta\|v\|_{H^{1}\left(\Omega^{\eta}\right)} .
$$


Proof of Lemma 3.2. The proof for $s=0$ is trivial (see Lemma 3.1). For positive $s$, we consider two cases. First we examine triangles whose boundaries do not intersect the boundary of any triangle in $\mathcal{T}_{1}$. We shall denote this set by $\tau \cap \mathcal{T}_{1}=\emptyset$ and the remaining set of triangles by $\tau \cap \mathcal{T}_{1} \neq \emptyset$.

Let $\phi_{i}$ be the nodal basis function in the space $V_{k}$ associated with the node $x_{i}^{k}$. Assume that $x_{i}^{k}$ does not lie on the boundary of any triangle $\tau \in \mathcal{T}_{1}$. Because of the multilevel construction, the mesh $\mathcal{T}_{k}$ is symmetric with respect to reflection through the point $x_{i}^{k}$. It follows that the nodal basis function $\phi_{i}$, restricted to a line passing through $x_{i}^{k}$, is an even function with respect to $x_{i}^{k}$. Let $x_{i}^{k}=\left(p_{1}, p_{2}\right)$. Then, both of the functions $x-p_{1}$ and $y-p_{2}$ are odd on each such line. Consequently,

$$
\left(x-p_{1}, \phi_{i}\right)=\left(y-p_{2}, \phi_{i}\right)=0 .
$$

Thus, it follows from Remark 3.1 that $\widetilde{Q}_{k} \widetilde{u}\left(x_{i}^{k}\right)=\widetilde{u}\left(x_{i}^{k}\right)$ for any linear function $\widetilde{u}$.

Let $\tau$ be a triangle whose boundary does not intersect the boundary of any triangle of $\mathcal{T}_{1}$. Applying the above argument to each node of $\tau$ shows that $\widetilde{Q}_{k} \widetilde{u}=\widetilde{u}$ on $\tau$ for any linear function $\widetilde{u}$. Let $\widetilde{\tau}$ be as in Lemma 3.1. Given $u \in H^{2}(\widetilde{\tau})$, let $\widetilde{u}$ be the linear function satisfying (3.2). As in the proof of Lemma 3.1, we get

$$
\left\|u-\widetilde{Q}_{k} u\right\|_{L^{2}(\tau)}=\left\|u-\widetilde{Q}_{\widetilde{\tau}} u\right\|_{L^{2}(\tau)} \leq C\|u-\widetilde{u}\|_{L^{2}(\widetilde{\tau})} \leq C h_{k}^{s}\|u\|_{H^{s}(\widetilde{\tau})}
$$

for $s=0,1,2$. Summing the above inequality and interpolating gives

$$
\left(\sum_{\tau \cap \mathcal{T}_{1}=\emptyset}\left\|u-\widetilde{Q}_{k} u\right\|_{L^{2}(\tau)}^{2}\right)^{1 / 2} \leq C h_{k}^{s}\|u\|_{H^{s}(\Omega)}
$$

for $s \in[0,2]$.

We next consider the case when $\tau$ intersects an edge in the triangulation $\mathcal{T}_{1}$. Suppose that $\tau$ intersects $\Gamma_{D}$. We clearly have that

$$
\left\|\widetilde{Q}_{k} u\right\|_{L^{2}(\tau)} \leq C\|u\|_{L^{2}(\widetilde{\tau})} .
$$

Thus,

$$
\left\|u-\widetilde{Q}_{k} u\right\|_{L^{2}(\tau)} \leq C\|u\|_{L^{2}(\widetilde{\tau})} .
$$

Summing the above inequality and applying (3.7) gives

$$
\left(\sum_{\tau \cap \Gamma_{D} \neq \emptyset}\left\|u-\widetilde{Q}_{k} u\right\|_{L^{2}(\tau)}^{2}\right)^{1 / 2} \leq C h_{k}\|u\|_{H^{1}\left(\Omega^{2 h_{k}}\right)} .
$$

Finally, we consider the case when $\tau$ intersects an edge in the triangulation $\mathcal{T}_{1}$ and does not intersect $\Gamma_{D}$. By Remark 3.1 and Lemma 3.1

$$
\left\|u-\widetilde{Q}_{k} u\right\|_{L^{2}(\tau)} \leq C h_{k}\|u\|_{H^{1}(\widetilde{\tau})} .
$$

Summing the above inequality and using (3.10) gives

$$
\left(\sum_{\tau \cap \mathcal{T}_{1} \neq \emptyset}\left\|u-\widetilde{Q}_{k} u\right\|_{L^{2}(\tau)}^{2}\right)^{1 / 2} \leq C h_{k}\|u\|_{H^{1}\left(E^{2 h_{k}}\right)} .
$$

Here $E^{2 h_{k}}$ denotes the strip of width $\mathcal{O}\left(2 h_{k}\right)$ around all element edges from the initial triangulation $\mathcal{T}_{1}$. 
The lemma for $s=1$ follows by combining (3.8) and (3.11). The result for $s \in(0,1)$ follows by interpolation. For $1<s<3 / 2$, (3.6) and (3.11) imply

$$
\left(\sum_{\tau \cap \mathcal{T}_{1} \neq \emptyset}\left\|u-\widetilde{Q}_{k} u\right\|_{L^{2}(\tau)}^{2}\right)^{1 / 2} \leq C h_{k}^{s}\|u\|_{H^{s}(\Omega)} .
$$

The lemma for $1<s<3 / 2$ follows by combining the above inequality with 3.8. This completes the proof of the lemma.

Remark 3.2. We can extend these arguments to the case when $V_{k}$ consists of piecewise quadratic functions with respect to the $k$ 'th triangulation. Again $\left\{\phi_{k}\right\}$ denotes the nodal basis for $V_{k}$. Then $\tilde{Q}_{k}$ defined by (3.5) satisfies Lemma [3.2. The proof is identical to the case of linears.

3.3. The coercivity estimate. We next show that the coercivity estimate (2.4) holds for $\widetilde{Q}_{k}$. Actually, we only require that the triangulation $\mathcal{T}_{h}$ be locally quasiuniform. We assume that $\Gamma_{D}$ aligns with this triangulation and let $V_{h}$ be the functions which are piecewise linear with respect to this triangulation, continuous on $\Omega$ and vanish on $\Gamma_{D}$. We consider the linear operator $\widetilde{Q}_{h}$ defined analogously to $\widetilde{Q}_{k}$ in (3.5) and show that

$$
\|v\|^{2} \leq C\left(\widetilde{Q}_{h} v, v\right), \quad \text { for all } v \in V_{h} .
$$

The constant $C$ above only depends on the quasi-uniformity constant (or minimal angle).

Let $\left\{x_{i}\right\}$ for $i=1, \ldots, m$ be the nodes of the triangulation and $\left\{\phi_{i}\right\}$ the corresponding nodal basis functions. The mesh is quasi-uniform, so for each $\phi_{i}$, there is a parameter $h_{i}$ such that

$$
h_{\tau} \simeq h_{i}
$$

for all triangles $\tau$ which have the node $x_{i}$ as a vertex. Here we define $a \simeq b$ to mean that

$$
a \leq C b \quad \text { and } \quad b \leq C a
$$

with constant $C$ independent of the triangulation. It is well known that

$$
(v, v) \simeq \sum_{\tau \in \mathcal{T}_{h}} h_{\tau}^{2} \sum_{x_{l} \in \tau} v\left(x_{l}\right)^{2}, \quad \text { for all } v \in V_{h} .
$$

It follows from 3.12 that

$$
(v, v) \simeq \sum_{i=1}^{m} h_{i}^{2} v\left(x_{i}\right)^{2}, \quad \text { for all } v \in V_{h} .
$$

We can now prove the coercivity estimate. This result was essentially given in [3] for the case of a globally quasi-uniform triangulation.

Lemma 3.4. Assume that the mesh $\mathcal{T}_{h}$ is locally quasi-uniform. There is a constant $C$, depending only on the quasi-uniformity condition, such that

$$
C^{-1}(v, v) \leq\left(\widetilde{Q}_{h} v, v\right) \leq C(v, v), \quad \text { for all } v \in V_{h} .
$$


Proof. Let $G$ be the Gram matrix, i.e.

$$
G_{i j}=\left(\phi_{i}, \phi_{j}\right), \quad i, j=1, \ldots, m,
$$

and let $D$ be the diagonal matrix with entries $D_{i i}=h_{i}^{2}$. Let $v$ be in $V_{h}$, and let $w$ be the coefficient vector satisfying

$$
v=\sum_{i=1}^{m} w_{i} \phi_{i}
$$

Note that (3.14) can be rewritten as

$$
C^{-1}((G w, w)) \leq((D w, w)) \leq C((G w, w)), \quad \text { for all } w \in R^{m} .
$$

Here $((\cdot, \cdot))$ denotes the inner product on $R^{m}$. This is equivalent to

$$
C^{-1}\left(\left(D^{-1} G w, G w\right)\right) \leq((G w, w)) \leq C\left(\left(D^{-1} G w, G w\right)\right), \quad \text { for all } w \in R^{m} .
$$

Since

$$
\left(1, \phi_{i}\right) \simeq h_{i}^{2}
$$

it follows that

$$
\begin{aligned}
\left(\widetilde{Q}_{h} v, v\right) & =\sum_{i=1}^{m} \frac{\left(v, \phi_{i}\right)^{2}}{\left(1, \phi_{i}\right)}=\sum_{i=1}^{m} \frac{\left((G w)_{i}\right)^{2}}{\left(1, \phi_{i}\right)} \\
& \simeq\left(\left(D^{-1} G w, G w\right)\right) \simeq((G w, w))=(v, v) .
\end{aligned}
$$

This completes the proof of the lemma.

\section{Applications}

In this section, we apply some of the above results. As we have seen in the previous section, the operator $\widetilde{Q}_{k}$ satisfies the approximation and coercivity estimates required for application of the abstract results. Throughout this section, we assume that $V_{1} \subset V_{2} \subset \ldots$ is a sequence of nested piecewise linear and continuous multilevel spaces, as described earlier. We take $V=L^{2}(\Omega)$, and $(\cdot, \cdot)$ is the corresponding inner product. With a slight abuse of notation we also use $(\cdot, \cdot)$ to denote the obvious duality pairing.

Remark 4.1. Since $V_{k} \subset H^{s}(\Omega)$, for $0 \leq s<3 / 2, Q_{k}$ and $\widetilde{Q}_{k}$ extend naturally to all of $H^{-s}(\Omega)$. Let $-3 / 2<s<3 / 2$ and define $\mathcal{A}^{s}$ as in (1.2). It is known that the norm $\left(\mathcal{A}^{s} u, u\right)^{1 / 2}$ is equivalent to $\|\cdot\|_{H^{s}(\Omega)}$; cf. [14].

Fix $\gamma<3 / 2$. By Lemma 3.2, the triangle inequality and well known properties of $Q_{k}$

$$
\left\|\left(Q_{k}-\widetilde{Q}_{k}\right) u\right\|_{L^{2}(\Omega)} \leq C \theta_{k}^{-1 / 2}\|u\|_{H^{\gamma}(\Omega)},
$$

where $\theta_{k}=h_{k}^{-2 \gamma}$. Let $s<\gamma$ and set $\mu_{k}=h_{k}^{-2 s}$. Then,

$$
\ell_{k, j}=\left(\frac{h_{k}}{h_{j}}\right)^{\gamma-s}
$$

decays exponentially as a function of $k-j$. An elementary computation gives that

$$
\|\mathcal{L}\| \leq C_{\mathcal{L}}=\left(1-\left(\frac{1}{2}\right)^{\gamma-s}\right)^{-1} .
$$

The next theorem immediately follows from Remark 4.1, Remark 2.2 and Theorem 2.1. 
Theorem 4.1. Let $-3 / 2<s<3 / 2$. Then $\left(\widetilde{\mathcal{A}}^{(s)} u, u\right)^{1 / 2}$ provides a norm on $H_{D}^{s}(\Omega)$ which is equivalent to the usual Sobolev norm. Here

$$
\widetilde{\mathcal{A}}^{(s)} u=\sum_{k=1}^{\infty} h_{k}^{-2 s}\left(\widetilde{Q}_{k}-\widetilde{Q}_{k-1}\right)^{2} u
$$

4.1. A preconditioning example. We consider applying the earlier results to develop a preconditioner for an example involving a pseudo-differential operator of order minus one. The canonical example of such an application is associated with a form

$$
\mathcal{V}(u, v)=\int_{\Omega} \int_{\Omega} \frac{u\left(s_{1}\right) v\left(s_{2}\right)}{\left|s_{1}-s_{2}\right|} d s_{1} d s_{2} .
$$

For this application, $\Gamma_{D}$ is empty and we seek preconditioners for the problem: Find $U \in V_{J}$ satisfying

$$
\mathcal{V}(U, \phi)=F(\phi) \quad \text { for all } \phi \in V_{J} .
$$

Here $F$ is a given functional. It is shown in [2] that

$$
\mathcal{V}(u, u) \simeq\|u\|_{H^{-1 / 2}(\Omega)}^{2} \quad \text { for all } u \in V_{J} .
$$

It is convenient to consider the problem of preconditioning in terms of operators. Specifically, let $\mathcal{V}: V_{J} \rightarrow V_{J}$ be defined by

$$
(\mathcal{V} v, w)=\mathcal{V}(v, w) \quad \text { for all } v, w \in V_{J} .
$$

We shall see that $\widetilde{\mathcal{A}}_{J}^{(1 / 2)}$ defined by

$$
\widetilde{\mathcal{A}}_{J}^{(1 / 2)}=\sum_{k=1}^{J} h_{k}^{-1}\left(\widetilde{Q}_{k}-\widetilde{Q}_{k-1}\right)^{2}
$$

provides a computationally efficient preconditioner for $\mathcal{V}$. Indeed, by Theorem 2.1

$$
\left(\widetilde{\mathcal{A}}_{J}^{(1 / 2)} u, u\right) \simeq\left(\mathcal{A}^{1 / 2} u, u\right) \quad \text { for all } u \in V_{J} .
$$

Applying Remark 4.1 and (4.1) gives us

$$
\left(\mathcal{V} \mathcal{A}^{1 / 2} u, \mathcal{A}^{1 / 2} u\right) \simeq\left(\mathcal{A}^{-1 / 2} \mathcal{A}^{1 / 2} u, \mathcal{A}^{1 / 2} u\right)=\left(u, \mathcal{A}^{1 / 2} u\right)
$$

for all $u \in V_{J}$. Thus, $\widetilde{\mathcal{A}}_{J}^{(1 / 2)} \mathcal{V}$ has a bounded spectral condition number.

It is easy to evaluate the action of $\widetilde{\mathcal{A}}_{J}^{(1 / 2)}$ in a preconditioned iteration procedure. For $k=1,2, \ldots, J$, let $\left\{\phi_{i}^{k}\right\}$ denote the nodal basis for $V_{k}$. In typical preconditioning applications, one is required to evaluate the action of the preconditioner on a function $v$ where only the quantities $\left\{\left(v, \phi_{i}^{J}\right)\right\}$ are known. One could, of course, compute $v$ from $\left\{\left(v, \phi_{i}^{J}\right)\right\}$, but this would require solving a Gram matrix problem. Our preconditioner avoids the Gram matrix problem. To evaluate the action of $\widetilde{Q}_{k}$, for $1 \leq k \leq J$, one is only required to take linear combinations of the quantities $\left\{\left(v, \phi_{i}^{\bar{k}}\right)\right\}$. Note that $\left(v, \phi_{i}^{k}\right)$ is a simple linear combination of $\left\{\left(v, \phi_{i}^{k+1}\right)\right\}$. Thus, we see that all of the $\widetilde{Q}_{k}$ 's can be computed efficiently (with work proportional to the number of unknowns on the finest level $J$ ) by a V-cycle-like algorithm. 
4.2. Examples involving sums of operators. We next consider preconditioning a sum of operators. The first example involves preconditioning the discrete systems which result from time stepping a parabolic initial value problem. The second example considers a Tikhonov regularization of a problem with noisy data.

Fully discrete time stepping schemes for parabolic problems often lead to problems of the form: Find $u \in S_{h}$ satisfying

$$
(u, \phi)+\epsilon D(u, \phi)=F(\phi) \quad \text { for all } \phi \in S_{h} .
$$

Here $D(\cdot, \cdot)$ denotes the Dirichlet form on $\Omega$ and $S_{h}$ is the finite element approximation. The parameter $\epsilon$ is related to the time step size and is often small. Assume that $S_{h}=V_{J}$, where $V_{J}$ is a multilevel approximation space as developed earlier. Let $\mu_{k}=1$ and $\hat{\mu}_{k}=h_{k}^{2}$, for $k=1,2, \ldots$ For convenience, we assume that $\Gamma_{D}$ is non-empty, so that $D(v, v) \simeq\|v\|_{1}^{2}$ for all $v \in H_{D}^{1}(\Omega)$. Then for $L_{J}$ and $\hat{L}_{J}$ defined respectively by (2.16) and (2.18), we have

$$
\left(L_{J} v, v\right) \simeq(v, v) \text { and }\left(\hat{L}_{J} v, v\right) \simeq D(v, v)
$$

for all $v \in V_{J}$. Applying Corollary 2.1 we see that the operator

$$
B_{J}=\sum_{k=1}^{J}\left(\mu_{k}^{-1}+\epsilon \hat{\mu}_{k}^{-1}\right)^{-1}\left(\widetilde{Q}_{k}-\widetilde{Q}_{k-1}\right)^{2}
$$

provides a uniform preconditioner for the discrete operator associated with (4.2). The resulting condition number for the preconditioned system can be bounded independently of the time step size $\epsilon$ and the number of levels $J$.

We next consider an example which results from Tikhonov regularization of a problem with noisy data. We consider approximating the solution of the problem

$$
T v=f,
$$

where $T$ denotes the inverse of the Laplacian and $f \in L^{2}(\Omega)$. This is replaced by the discrete problem

$$
T_{h} v=f_{h},
$$

where $T_{h}$ is the Galerkin solution operator, i.e., $T_{h} v=w$, where $w \in V_{J}$ satisfies

$$
D(w, \theta)=(v, \theta) \quad \text { for all } \theta \in V_{J}
$$

and $f_{h}$ is the $L^{2}(\Omega)$ orthogonal projection onto $V_{J}$. If it is known that $v$ is smooth but $f$ is noisy, better approximations result from regularization [11, [17. We consider the regularized solution $\widetilde{w} \in V_{J}$ satisfying

$$
\left(T_{h}+\alpha A_{h}\right) \widetilde{w}=f_{h} .
$$

Here $A_{h}: V_{J} \rightarrow V_{J}$ is defined by

$$
\left(A_{h} v, w\right)=D(v, w) \quad \text { for all } v, w \in V_{J} .
$$

The regularization parameter $\alpha$ is often small (see [17]), and can be chosen optimally in terms of the magnitude of the noise in $f$.

Preconditioners for the sum in (4.4) of the form of (4.3) result from the application of Corollary 2.1 In this case, $\mu_{k}=h_{h}^{-2}, \hat{\mu}_{k}=h_{k}^{2}$. The condition numbers for the resulting preconditioned systems can be bounded independently of the regularization parameter $\alpha$.

Preconditioners for systems like (4.4) are generally not easily developed. The problem is that the operator applied to the higher frequencies (depending on the 
size of $\alpha$ ) behaves like a differential operator, while on the lower frequencies it behaves like the inverse of a differential operator. This causes difficulty in most multilevel methods.

4.3. $H^{1}(\Omega)$ bounded extensions. As a final application, we consider the construction of $H^{1}(\Omega)$ bounded extensions. Such extensions are useful in development of domain decomposition preconditioners with inexact subdomain solves. The construction given here is essentially the same as that in [12. We include it here in detail as an application of Theorem 4.1.

With $\left\{V_{j}\right\}$ as above, let $\widetilde{V}_{k}$ (for $\left.k=1,2, \ldots, J\right)$ be the functions defined on $\partial \Omega$ which are restrictions of those in $V_{k}$. This gives a multilevel structure on the finest space $\tilde{V}_{J}$. These spaces inherit a nodal basis from the original nodal basis on $V_{k}$. The nodal basis function associated with a boundary node $x_{i}$ is just the restriction of the basis function for $V_{k}$ associated with $x_{i}$. Denoting this basis by $\left\{\psi_{i}^{k}\right\}$, we define

$$
\widetilde{q}_{k}(f)=\sum \frac{\left\langle f, \psi_{i}^{k}\right\rangle}{\left\langle 1, \psi_{i}^{k}\right\rangle} \psi_{i}^{k}
$$

The above sum is taken over the nodal basis elements for $\widetilde{V}_{k}$, and $\langle\cdot, \cdot\rangle$ denotes the $L^{2}(\partial \Omega)$ inner product. We note that it is known [14 that

$$
\|\theta\|_{H^{1 / 2}(\partial \Omega)}^{2} \simeq \sum_{k=1}^{J} h_{k}^{-1}\left\|\left(q_{k}-q_{k-1}\right) \theta\right\|_{L^{2}(\partial \Omega)}^{2},
$$

where $q_{k}$ denotes the $L^{2}$-projection onto $\tilde{V}_{k}$. It is easy to see that Theorem 4.1 holds for these spaces. Thus

$$
\|\theta\|_{H^{1 / 2}(\partial \Omega)}^{2} \simeq \sum_{k=1}^{J} h_{k}^{-1}\left\|\left(\widetilde{q}_{k}-\widetilde{q}_{k-1}\right) \theta\right\|_{L^{2}(\partial \Omega)}^{2},
$$

with $\widetilde{q}_{J} \theta=\theta$ and $\widetilde{q}_{0} \theta=0$.

Now, given a function $\theta \in \widetilde{V}_{J}$, we define $E_{J} \theta \in V_{J}$ by $E_{J} \theta=\sum_{k=1}^{J} \omega_{k}$, with $\omega_{k}$ defined as follows. Let $\bar{\theta}$ be the mean value of $\theta$ on $\partial \Omega$. Then $\omega_{1}$ is the function in $V_{1}$ satisfying

$$
\omega_{1}\left(x_{i}\right)=\left\{\begin{array}{cl}
\widetilde{q}_{1}\left(x_{i}\right) & \text { if } x_{i} \text { is a node of } V_{1} \text { on } \partial \Omega, \\
\bar{\theta} & \text { if } x_{i} \text { is a node of } V_{1} \text { in the interior of } \Omega .
\end{array}\right.
$$

For $J \geq k>1, \omega_{k}$ is the function in $V_{k}$ satisfying

$$
\omega_{k}\left(x_{i}\right)=\left\{\begin{array}{cl}
{\left[\widetilde{q}_{k} \theta-\widetilde{q}_{k-1} \theta\right]\left(x_{i}\right)} & \text { if } x_{i} \text { is a node of } V_{k} \text { on } \partial \Omega, \\
0 & \text { if } x_{i} \text { is a node of } V_{k} \text { in the interior of } \Omega .
\end{array}\right.
$$

Note that $E_{J} \theta=\theta$ on $\partial \Omega$, so that $E_{J}$ is an extension operator.

Recall that $|\cdot|_{H^{1}(\Omega)}$ denotes the semi-norm on $H^{1}(\Omega)$. Then

$$
\left|E_{J} \theta\right|_{H^{1}(\Omega)}=\left|E_{J} \theta-\bar{\theta}\right|_{H^{1}(\Omega)}=\left|E_{J}(\theta-\bar{\theta})\right|_{H^{1}(\Omega)} \leq\left\|E_{J}(\theta-\bar{\theta})\right\|_{H^{1}(\Omega)} .
$$

We now use the following well known multilevel characterization of the $H^{1}(\Omega)$ norm on $V_{J}$ :

$$
\|v\|_{H^{1}(\Omega)}^{2} \simeq \inf \sum_{k=1}^{J} h_{k}^{-2}\left\|v_{k}\right\|_{L^{2}(\Omega)}^{2}
$$


where the infimum is taken over all splittings $v=\sum_{k=1}^{J} v_{k}$ with $v_{k} \in V_{k}$. Applying this with $v=E_{J}(\theta-\bar{\theta})=\left(\omega_{1}-\bar{\theta}\right)+\sum_{k=2}^{J} \omega_{k}$ and using (4.5), we conclude that

$$
\begin{aligned}
& \left\|E_{J}(\theta-\bar{\theta})\right\|_{H^{1}(\Omega)}^{2} \leq C\left[\sum_{k=2}^{J} h_{k}^{-2}\left\|\omega_{k}\right\|_{L^{2}(\Omega)}^{2}+h_{1}^{-2}\left\|\omega_{1}-\bar{\theta}\right\|_{L^{2}(\Omega)}^{2}\right] \\
& \quad \leq C \sum_{k=1}^{J} h_{k}^{-1}\left\|\left(\widetilde{q}_{k}-\widetilde{q}_{k-1}\right)(\theta-\bar{\theta})\right\|_{L^{2}(\partial \Omega)}^{2} \leq C\|\theta-\bar{\theta}\|_{H^{1 / 2}(\partial \Omega)}^{2} \leq C|\theta|_{H^{1 / 2}(\partial \Omega)}^{2},
\end{aligned}
$$

where $|\cdot|_{H^{1 / 2}(\partial \Omega)}$ denotes the $H^{1 / 2}(\partial \Omega)$ semi-norm. Thus we see that

$$
\left|E_{J} \theta\right|_{H^{1}(\Omega)} \leq C|\theta|_{H^{1 / 2}(\partial \Omega)} \text {. }
$$

This type of bounded extension operator is precisely what is required for the development of non-overlapping domain decomposition algorithms which do not involve the exact solution of subproblems.

\section{Numerical Results}

We present the results of some numerical experiments using the operator $\widetilde{\mathcal{A}}_{J}$ defined by (1.4) applied as a preconditioner for various discrete differential and pseudo-differential operators. The first example is a standard one and involves preconditioning the finite element discretization of a second order problem. Although there are many methods available for this problem, we consider it here since it is the best-studied problem. The second problem involves using (4.3) to precondition a sum of operators similar to (4.4).

We start with preconditioning the Laplace operator with Neumann boundary conditions. To make this problem definite, we consider both the finite element operator and the preconditioner on the $L^{2}$-orthogonal complement of the one dimensional subspace of constants. The finite element space $\widetilde{V}_{k}$ consists of piecewise linear functions defined with respect to a uniform triangulation of the square which results when an equally spaced $n_{k} \times n_{k}$ mesh of smaller squares is partitioned into triangles by connecting the lower left and upper right hand vertices. Here we take $n_{k}=2^{k}$ and define $\widetilde{V}_{k}$ to be the functions in $V_{k}$ which are orthogonal to constants. Let $\widetilde{Q}_{k}$ be defined as in (3.5) with respect to the space $\widetilde{V}_{k}$.

The BPX-like preconditioner

$$
\widetilde{B}_{J}=\sum_{k=0}^{J} h_{k}^{2} \widetilde{Q}_{k}
$$

provides a uniform preconditioner for the Galerkin discretization of the problem

$$
\begin{aligned}
& u-\Delta u=f \quad \text { in } \Omega \text {, } \\
& \frac{\partial u}{\partial n}=0 \quad \text { on } \partial \Omega,
\end{aligned}
$$

using the approximation subspace $\widetilde{V}_{J}$. Here $n$ denotes the outward normal direction on $\partial \Omega$. Let $Q_{J}$ denote the $L^{2}(\Omega)$-orthogonal projector onto $V_{J}$. The operator $Q_{J} \widetilde{B}_{J}$ is symmetric and positive definite on $V_{J}$ and is a uniform preconditioner for the Galerkin approximation $A_{h}$ to

$$
\begin{aligned}
-\Delta u=f & & \text { in } \Omega, \\
\frac{\partial u}{\partial n}=0 & & \text { on } \partial \Omega,
\end{aligned}
$$

based on the approximation subspace $V_{J}$. 
TABLE 1. Condition numbers for BPX and $B_{J}$ applied to (5.1).

\begin{tabular}{|c|c|c|}
\hline$h_{J}$ & $K(\mathrm{BPX})$ & $K\left(B_{J}\right)$ \\
\hline $1 / 8$ & 9.8 & 5.9 \\
$1 / 16$ & 11.3 & 7.3 \\
$1 / 32$ & 12.1 & 8.4 \\
$1 / 64$ & 12.9 & 9.1 \\
$1 / 128$ & 13.4 & 9.6 \\
$1 / 256$ & 13.7 & 10.1 \\
$1 / 512$ & 13.9 & 10.4 \\
\hline
\end{tabular}

Table 1 reports the condition number of $Q_{J} \widetilde{B}_{J}$ as a function of $h_{J}$ for both the BPX preconditioner and the preconditioner defined by

$$
B_{J}=\sum_{k=2}^{J} h_{k}^{2}\left(\widetilde{Q}_{k}-\widetilde{Q}_{k-1}\right)^{2} .
$$

Note that $B_{J}$ annihilates constants, since we have omitted the $k=1$ term in the above sum. We see that in this simple case, the new preconditioner is somewhat better than the BPX-like preconditioner although it is slightly more complicated to apply.

The second example illustrates the performance of a preconditioner of the form of (2.19) applied to the problem $\alpha A_{h}+T_{h}$. Specifically,

$$
B_{J}=\sum_{k=2}^{J}\left(\alpha h_{k}^{-2}+h_{k}^{2}\right)^{-1}\left(\widetilde{Q}_{k}-\widetilde{Q}_{k-1}\right)^{2} .
$$

The operator $T_{h}$ used here is an operator which is spectrally very close to $A_{h}^{-1}$. It is defined to be the solution operator of the problem: Find $w \in V_{J}$ satisfying

$$
D(w, \phi)=(v, \phi)_{*} \quad \text { for all } \phi \in V_{J},
$$

i.e., $T_{h} v=w$. The inner product $(\cdot, \cdot)_{*}$ is a minor perturbation of $(\cdot, \cdot)$ which makes the computation of $T_{h}$ feasible via the fast Fourier transform.

We report the condition numbers for $h_{J}$ between $1 / 8$ and $1 / 512$ and for $\alpha=h_{J}^{\gamma}$, $\gamma=0,1,2,4$ (see Table 2). Although there are some values of $\gamma$ for which $\alpha A_{h}+T_{h}$ can be preconditioned by other methods, (5.2) provides good preconditioning for all choices of the parameter as guaranteed by Corollary 2.1. Note in particular the examples $\gamma=1$ and $\gamma=2$. For these cases, the operator behaves like a differential operator on the higher frequencies and like a pseudo-differential operator of negative order on the lower frequencies. As far as we know, methods for preconditioning such an operator are not available in the literature.

TABLE 2. Condition numbers when preconditioning $h_{J}^{\gamma} A_{h}+T_{h}$.

\begin{tabular}{|c|c|c|c|c|}
\hline$h_{J}$ & $\gamma=0$ & $\gamma=1$ & $\gamma=2$ & $\gamma=4$ \\
\hline $1 / 8$ & 5.9 & 12.0 & 21.6 & 14.9 \\
$1 / 16$ & 7.2 & 17.6 & 35.8 & 15.5 \\
$1 / 32$ & 8.1 & 24.3 & 43.2 & 16.4 \\
$1 / 64$ & 8.9 & 34.2 & 46.8 & 16.9 \\
$1 / 128$ & 9.5 & 45.9 & 50.8 & 17.1 \\
$1 / 256$ & 10.0 & 55.2 & 54.5 & 17.3 \\
$1 / 512$ & 10.4 & 61.1 & 57.6 & 17.4 \\
\hline
\end{tabular}




\section{REFERENCES}

[1] J. H. Bramble and J. E. Pasciak New estimates for multigrid algorithms including the V-cycle Math. Comp. 60(1993),447-471. MR 94a:65064]

[2] J.H. Bramble, Z. Leyk, and J.E. Pasciak, Iterative schemes for non-symmetric and indefinite elliptic boundary value problems, Math. Comp. 60 (1993) 1-22. MR 93d:65034

[3] J. H. Bramble, J. E. Pasciak and J. Xu, Parallel multilevel preconditioners, Math. Comp. 55(1990), 1-22. MR 90k:65170

[4] J. H. Bramble and P. S. Vassilevski, Wavelet-like extension operators in interface domain decomposition methods, (unpublished manuscript) 1997.

[5] J. M. Carnicer, W. Dahmen, and J. M. Peña, Local decompositions of refinable spaces and wavelets, Appl. Comp. Harm. Anal. 3(1996), 127-153. MR 97f:42050

[6] P.G. Ciarlet, The Finite Element Method for Elliptic Problems, North-Holland,New York, 1978. MR 58:25001

[7] P. Grisvard, Elliptic Problems in Nonsmooth Domains, Pitman, Boston, 1985. MR 86m:35004

[8] G. Haase, U. Langer, A. Meyer, and S. V. Nepomnyaschikh, Hierarchical extension operators and local multigrid methods in domain decomposition preconditioners, East-West J. Numer. Math. 2(1994), 173-193. MR 95g:65171

[9] U. Kotyczka and P. Oswald, Piecewise linear prewavelets of small support, in: Approximation Theory VIII, vol. 2 (wavelets and multilevel approximation), C. Chui, L.L. Schumaker, eds., World Scientific, Singapore, 1995, 235-242. MR 98e:42035

[10] R. Lorentz and P. Oswald, Constructing economical Riesz bases for Sobolev spaces, Proceedings of the Domain Decomposition Conference held in Bergen, Norway, June 3-8, 1996.

[11] F. Natterer, Error bounds for Tikhonov regularization in Hilbert scales, Appl. Anal. 18(1984), 29-37. MR 86e:65081]

[12] S. V. Nepomnyaschikh, Optimal multilevel extension operators, Report SPC 95-3, Jan, 1995, Technische Universität Chemnitz-Zwickau, Germany.

[13] P. Oswald, On discrete norm estimates related to multilevel preconditioners in the finite element method, Constructive Theory of Functions, Proc. Int. Conf. Varna 1992, Bulg. Acad. Sci., Sofia, 1992, 203-214.

[14] P. Oswald, Multilevel Finite Element Approximation, Theory and Applications B.G. Teubner Stuttgart, Germany 1994. MR 95k:65110

[15] R. Stevenson, A robust hierarchical basis preconditioner on general meshes, Numer. Math. 78 (1997), 269-303. CMP 98:05

[16] R. Stevenson, Piecewise linear (pre-) wavelets on non-uniform meshes, Report \# 9701, Department of Mathematics, University of Nijmegen, Nijmegen, The Netherlands, 1997.

[17] U. Tautenhahn, Error estimates for regularization methods in Hilbert scales, SIAM J. Numer. Anal. 33(1996), 2120-2130.

[18] P. S. Vassilevski and J. Wang, Stabilizing the hierarchical basis by approximate wavelets, I: Theory, Numer. Linear Alg. Appl. 4(1997), 103-126. MR 98c:65197

[19] P. S. Vassilevski and J. Wang, Stabilizing the hierarchical basis by approximate wavelets, II: Implementation and numerical experiments, SIAM J. Sci. Comput. 20 (1999), 490-514. CMP 98:17

[20] X. Zhang, Multi-level Additive Schwarz Methods, Courant Inst. Math. Sci., Dept. Comp. Sci. Rep. 1991.

Department of Mathematics, Texas A \& M University, College Station, Texas 77843

E-mail address: bramble@math.tamu.edu

E-mail address: pasciak@math.tamu.edu

Central laboratory of Parallel Processing, Bulgarian Academy of Sciences, "Acad. G. Bontchev" Street, Block 25 A, 1113 Sofia, Bulgaria

E-mail address: panayot@iscbg.acad.bg

Current address: Center for Applied Scientific Computing, Lawrence Livermore National Laboratory, P. O. Box 808, L-560, Livermore, CA 94551, U.S.A.

E-mail address: panayot@llnl.gov 